RAD Conference Proceedings, vol. 2, pp. 228-230, 2017

www.rad-proceedings.org

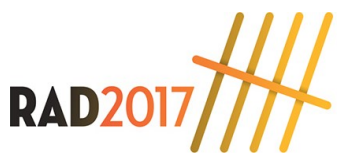

\title{
THE IMPACT OF SMOKING ON THE HEALTH OF PERIODONTAL TISSUE
}

\author{
Ilma Robo' ${ }^{*}$, Saimir Heta², Panajot Papa3, Edlira Sadiku4, Nevila Alliu5
}

${ }^{1}$ Head of Department of Periodontology, Albanian University, Tirana, Albania

${ }^{2}$ Hospital Center, Pediatric Surgery, Surgeon Pediatrician, Tirana, Albania

3Faculty of Medical Sciences, University of Elbasan, Albania

4Private Clinic, Tirana, Albania

5University Hospital Center, Biochemical Laboratory, Tirana, Albania

\begin{abstract}
The aim is to emphasize the notions: determinant indicator and predictor of risk factors for periodontal pathologies, and to show the interconnection logic, analyzing the effects of one of the risk factors, in this case of smoking, mainly on the amount of gingival fluid. For achieving this, measurements were carried out, in mm of the wet amount of adsorbent placed in sulcus, before and 30 minutes after smoking; held in sulcus, for 3 minutes. This procedure was repeated several times. In the end, we organized the summary table, to show the interconnection of concepts about periodontal risk. What represents the human body consists of the host which is subjected to the action of oral bacterial flora and this interaction extends in time, where the personal diet of the patient operates as mechanical element that cleans the surfaces of the tooth. It is noted that the change in wetting of the adsorbent was only 1 or 0.5 millimeters, within 3 minutes time that was held inside the sulcus. Small changes, but in total for the whole mouth, these are with great action for the oral hygiene. Once this $0.5 \mathrm{~mm}$ of humidity is produced in 6 different points, around the tooth, and for 32 teeth simultaneously, the cleaning action of gingival fluid is more effective. Smoking not only increases the amount of crevicular fluid, but it also promotes the destruction of the bone, whose clinical signs are visible to the naked eye.
\end{abstract}

Key words: Smoking, determinants, forecasters, indicators, crevicular fluid

DOI: $10.21175 /$ RadProc.2017.47

\section{INTRODUCTION}

Smoking is the most controversial element, serving as a predisposing factor to a variety of diseases of the body, and among them, to oral diseases. Whenever it acts, smoking has a negative effect to oral structures, particularly on mucosal tissue. The normal mucosal tissue has the lifecycle that is influenced by interventions of risk factors, interventions that occur once in life, and which operate continuously or operate systematically, whereas in terms of frequency, they act on regular or irregular rhythm [1]. Smoking, diabetes and bacterial plaque are elements that can be analyzed in longitudinal studies and with cross-sectional ones, as they estimate the risk factor present in the oral cavity. If time has null effect, we can check the effect of smoking in mucosal tissues at different ages, having constant genetic factors, gender, socio-economic and psychological effects of stress. Under the effect of smoking, we analyze the determinants of risk and risk indicators. Identification of the risk factors as not editable, while indicators of risk, as possible factors, contain high risk, but cannot be the cause of the real disease [1].

*ilmarobo@yahoo.com
Studies on the role of smoking to periodontal status have started in the years 1980-1990. Cross-sectional controls have proved that the incidence of gingivitis in smoking patients is not at a very serious grade, compared to the control group of non-smokers.

Based on studies, it is noted that smoking is associated with less bleeding and less gingival pockets depths, compared with non-smoker patients [2]. Most studies around the intersection of smoking and periodontal illnesses have supported the fact that tobacco negatively affects periodontal tissues, though various writers have failed to show this exact connection [2]. Very little research has focused on whether these periodontal effects act just like men and women [3].

\section{MATERIALS AND METHODS}

Normally, we expect that during the examination of smokers bleeding gingiva will appear, high amount of bacterial plaque and possibly painted with characteristic pigments of nicotine, and in fact, the smoking patient appears with regular oral hygiene, may suffer from coloration of the surface, and with minimum oral plaque. Our study is intended to 
examine the effect of smoking on periodontal tissues, especially on junctional epithelium, which serves as the basis of gingival sulcus. On the other hand, in this study we tend to emphasize the notions of determinant, indicator and predictor of risk factors for periodontal disease. We are trying to show their logical interconnection, analyzing the effects of one of the risk factors, in this case, smoking.

As we know, one of the ways of collection of crevicular fluid is through the adsorbents used in endodontic treatment. For simplicity of work, the territory of the right central in maxilla was used. Setting cotton roles, before initiating the procedure, is necessary for the achievement of dryness, to protect teeth from the wetting of the inside of the lip. With tweezers, place the absorbent inside the sulcus, while with the ruler emulate the wet length of absorbent.

We first performed measurements immediately after smoking; we decided the adsorbent in sulcus, for 3 minutes. Through ruler, we emulate the wet length of adsorbent. After 30 minutes, we again measured the amount of fluid in the same way, but in this case before smoking. Later, this procedure was repeated several times. In this study is calculated the weighted average.

The criterion on which the study was based is the fact that the amount of gingival fluid varies depending on the rate of daily smoking. It is known that it boosts production of gingival fluid according to a daily rate, determined by smokers themselves. Could this effect be exploitable in periodontal therapeutic methods?

\section{RESULTS}

The human body may represent a host organism that stands under the action of oral bacterial flora. This lasting action in time, where the mechanical elements of the individual diet of personal prescription can inhibit the adhesion of bacterial plaque, has an irreplaceable role.

These elements may still have a stimulant role, causing the periodontal disease, requiring the involvement of smoking, how addicted the patient is, diabetes as a systemic disease, and plaque, the presence of which indicates the lack of oral hygiene, culminating in typical clinical statuses of periodontal pathologies in oral cavities.

Following this logic, we started with the collection of data from the literature, in order to create the existing interconnection of determinants of risk, risk expectations and indicators of risk for periodontal disease, clearly stated in Table 1 . Based on the data collected from the literature, the smoking patients are less sensitive to bleeding on probing than the nonsmokers, followed by the fact that the plaque index shows no significant difference [3]. When the periodontal problems begin and where they show the elements of predictors of periodontal risk, the increases in depth of gingival sulcus, the gingival recession and the increase of the level of loss of gingival junction is observed. These are the elements with a more significant visible view to smoking patients, than to those who are non smokers [3].
Table 1. In the table, the way of analyzing the determinants, forecasters and indicators for risk is shown in longitudinal studies and cross-sectional ones

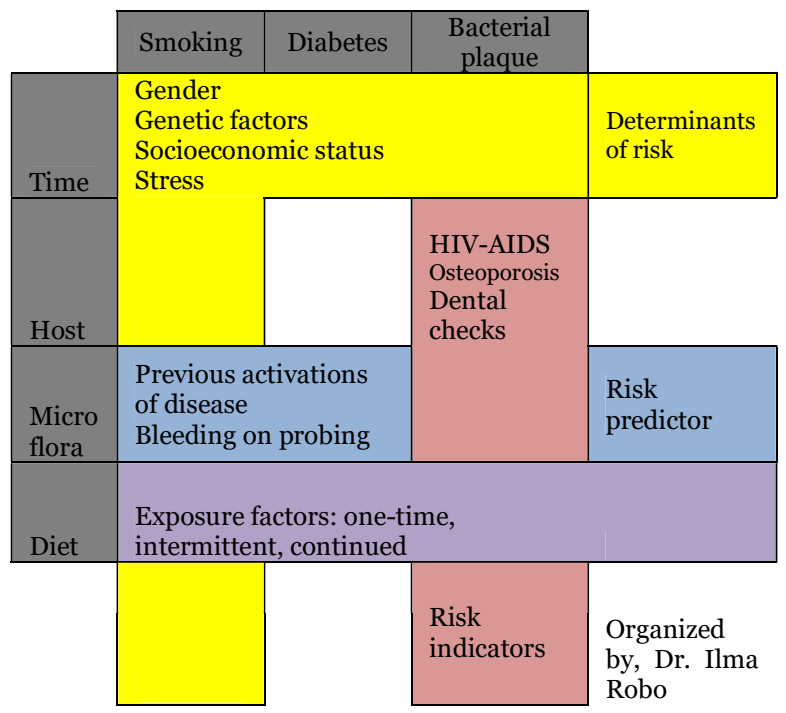

From the measurements that we obtained, it was observed that the amount of fluid increases under the influence of smoking, in parameters presented in Table 2.

Table 2. Amount of gingival fluid, measured in $\mathrm{mm}$ of wet adsorbent, positioned in gingival sulcus

\begin{tabular}{|c|c|c|c|}
\hline \multirow{7}{*}{$\begin{array}{c}\text { Number of } \\
\text { patients } \\
\text { according to } \\
\text { the category }\end{array}$} & $\begin{array}{c}\text { The amount } \\
\text { of fluid } \\
\text { "Before } \\
\text { smoking" }\end{array}$ & $\begin{array}{c}\text { The amount } \\
\text { of fluid } \\
\text { "After } \\
\text { smoking" }\end{array}$ \\
\cline { 2 - 4 } & 7 & $2 \mathrm{~mm}$ & $3 \mathrm{~mm}$ \\
\cline { 2 - 4 } & 6 & $3 \mathrm{~mm}$ & $4 \mathrm{~mm}$ \\
\cline { 2 - 4 } & 3 & $2.5 \mathrm{~mm}$ & $3 \mathrm{~mm}$ \\
\cline { 2 - 4 } & 5 & $1 \mathrm{~mm}$ & $2 \mathrm{~mm}$ \\
\cline { 2 - 4 } & 10 & $1.5 \mathrm{~mm}$ & $2 \mathrm{~mm}$ \\
\hline Total & 6 & $1.5 \mathrm{~mm}$ & $3 \mathrm{~mm}$ \\
\hline
\end{tabular}

The weighted average amount of gingival fluid before smoking is $1.9 \mathrm{~mm}$, and $3 \mathrm{~mm}$ after smoking. The difference found is $1 \mathrm{~mm}$ enhancement of gingival fluid.

If we carefully look at these data, it is noted that the change in wetting the adsorbent was only 1 or 0.5 millimeters, in 3 minutes. These are small changes, but having in mind the whole mouth, these are with great action for the oral hygiene. Once this $0.5 \mathrm{~mm}$ of humidity is produced in 6 different points, around the tooth, and for 32 teeth simultaneously, the cleaning action of gingival fluid is more effective.

\section{DISCUSSION}

The effect of smoking on periodontal tissues depends on the number of cigarettes consumed per day and the duration of addiction; the impact of the time element also causes the effect to be even more protracted [3].

Smoking is composed of nicotine, which on the one hand causes the constriction of blood vessels, weakens 
the body's defense against bacterial invasion caused by bacterial plaque but, on the other hand, from the other side effects [4], it increases the TNF-a, as well as the PGE-2 and metaloproteinaz-8. All these three elements, through their mechanisms of action, increase the destruction of one of the most important element of periodontal structures, such as bone.

In smoking patients, it significantly reduces the proportion of the secreted IgA at saliva and it decreases the number of $\mathrm{T}$ h-cells. On the other hand, since the involved TNF-a has a bacterial component, as the 37KDA protein, same as IL-1 and IL-6, then TNF-a, driven by the impact of nicotine, promotes the formation of IL-1 and IL-6 which are respectively in these host cells: IL-1 in PMN-s macrophages, in monocytes, and IL-6 in macrophages, infibroblasts and in epithelial cells.

The inflammation cells are also host cells. This means that they are stimulatory for the outset of the inflammation. Consequently, it is increasing the gingival fluid, because it is increasing the vascular permeability by the action of IL-1 and IL-6 [1]. This immediate and transitional increase of crevicular fluid we can be utilized during the local and systemic periodontal therapy, after the increasing of the time and of the amount of preparation that is in a direct contact with the tissue where the inflammation is. It is known that the crevicular fluid also flows in the systemic given tetracycline. By increasing the production of fluid, we can grow the frequency of crevicular spill of systemic given tetracycline and therefore we can grow the positive antibacterial effect against the bacterial oral flora. Then, can we use this effect of smoking in periodontal treatment of patients? Everything remains in the determination of the ratio advantage/disadvantage. These signs suggest that the effects will include medicines systematically given for periodontal reasons, even if smokers indicate and emphasize the importance of giving up smoking [5].

\section{CONCLUSIONS}

Smoking increases the crevicular amount of gingival fluid, but also promotes the destruction of bone, whose macroscopically clinical signs are more than visible. There is a logical connection between determinants, predictors and indicators for the risk of the periodontal diseases, whose analysis is carried out logically, with longitudinal studies and cross-sectional ones, without having to change one of the triggers of the disease and one of the risk factors of periodontal pathology.

\section{REFERENCES}

1. M. G. Newman, H. Takei, P. R. Klokkevold, F. A. Carranza, Clinical Periodontology, 10th ed., St. Louis (MO), USA: Saunders, 2014.

2. D. K. Gautam, V. Jindal, S. C. Gupta, A. Tuli, B. Kotwal, R. Thakur, "Effect of cigarette smoking on the periodontal health status: A comparative, cross sectional study," J. Indian Soc. Periodontol., vol. 15, no. 4, pp. 383-387, Oct. 2011.

DOI: $10.4103 / 0972-124 X .92575$

PMid: 22368364 PMCid: PMC 3283937

3. G. Calsina, J. M. Ramón, J. J. Echeverría, "Effects of smoking on periodontal tissues," Journal of Clinical Periodontology, vol. 29, no. 8, pp. 771-776, Aug. 2002; DOI: 10.1034/j.1600-051X.2002.290815.x PMid: 12390575

4. P. Obeid, P. Bercy, "Effects of smoking on periodontal health: a review," Adv. Ther., vol. 17, no. 5, pp. 230-237, Sep. 2000.

DOI: $10.1007 / \mathrm{BFO} 2853162$

PMid: 11186143

5. Periodontal Diseases - A Clinician's Guide, J. Manakil, Ed., Rijeka, Croatia: In Tech, 2012.

DOI: $10.5772 / 1274$ 